\section{Aortic root enlargement by Manouguian's technique}

\section{To the Editor:}

We read with interest the article by Kawachi, Tominaga, and Tokunaga ${ }^{1}$ titled "Eleven-Year Follow up Study of Aortic or Aortic-Mitral Anulus-Enlarging Procedure by Manouguian's Technique" and appreciate their results. However, our own experience is different from theirs.

Between June 1989 and September 1992, we have done aortic root enlargement by Manouguian's technique in 12 patients aged 20 to 51 years. One patient underwent aortic root enlargement as an emergency procedure for severe aortic regurgitation after balloon dilation of the aortic valve. In six patients, the anterior mitral leaflet was divided and a diamond-shaped Gelseal patch (gelatin-coated Dacron patch, Vascutek Inc., Inchinnan, Scotland) was sutured in the defect in the mitral leaflet. The edges of the Gelseal patch were buttressed by strips of pericardium as well. In the remaining six patients, a pericardial patch was used to bridge the defect in the mitral leaflet. Whenever the roof of the left atrium was opened, it was closed by a patch of pericardium.

A mechanical prosthetic valve, either St. Jude Medical (St. Jude Medical, Inc., St. Paul, Minn.) or Medtronic Hall (Medtronic, Inc., Minneapolis, Minn.), larger than the measured aortic root by either one or two sizes, was sewn into place with interrupted horizontal mattress sutures. None of the patients had any significant bleeding through the suture line. All the patients have been followed up for 6 months to 2 years and are doing well.

Unlike Kawachi, Tominaga, and Tokunaga, we used only mechanical valves. In addition, none of the patients had either mitral regurgitation or hemolysis. The patient-prosthesis mismatch or small-sized prosthesis can produce complications such as a significant gradient, left ventricular hypertrophy, thrombosis, and hemolysis. ${ }^{2}$ On the basis of our own experience and the report by Kawachi, Tominaga, and Tokunaga, we believe that aortic root enlargement can be done without much morbidity and the patient should be given the benefit of having a large prosthesis.

\section{Arun K. Bhutani, MCh Kesava Dev, $M C h$ C. H. Mallikharjuna Gupta, $M C h$ Kurudamannil A. Abraham, DM \\ Rajesh N. Desai, $M C h$ \\ Komarakshi R. Balakrishnan, $M C h$ Department of Cardiovascular Surgery Southern Railway Headquarters Hospital Perambur \\ Madras 600023, India}

\section{R EFERENCES}

1. Kawachi $Y$, Tominaga R, Tokunaga K. Eleven-year follow-up study of aortic or aortic-mitral anulus-enlarging procedure by Manouguian's technique. J THORAC CARDIOVASC SURG 1992;104:1259-63.

2. Rahimtoola $\mathrm{SH}$. The problem of valve prosthesis-patient mismatch. Circulation 1978;58:20-4.

\section{Type A aortic dissection: Management of brain malperfusion through retrograde cerebral perfusion}

To the Editor:

Antegrade organ malperfusion, and particularly cerebral malperfusion, is a life-threatening complication of acute aortic dissection that is due to an impaired flow in either the true or the false lumen. However, cerebral malperfusion can also occur at the time of surgical repair. This may be a consequence of the reversed flow from the femoral artery on initiation of cardiopulmonary bypass if no entry site is present in the ascending aorta; it may also appear when the intimal tear in the proximal ascending aorta is excluded at the moment the crossclamp is applied or when the surgical repair is completed. In the first two instances the problem is managed either by repositioning the arterial cannula into the true aortic lumen or fenestrating the membrane within the proximal $\operatorname{arch}^{1}$ or, whenever possible, by pharmacologically maintaining an adequate pressure as systemic hypothermia is induced. ${ }^{2}$ In the last case the problem is solved by reestablishing an antegrade perfusion via the ascending aortic graft. ${ }^{3}$ Herein we present a case of cerebral malperfusion that was successfully managed by selective retrograde cerebral perfusion through the right internal jugular vein.

A 56-year-old woman was admitted with unreceding chest pain. Echocardiography and magnetic resonance imaging studies showed a type A aortic dissection with the two lumina extending from the proximal ascending aorta through to the abdominal aorta, as well as severe aortic valve insufficiency. All peripheral pulses were present. The patient underwent urgent surgical treatment. The left femoral artery did not have evidence of dissection and was cannulated for cardiopulmonary bypass. The right atrium was cannulated for venous return. Cardiopulmonary bypass was started and, on occurrence of ventricular fibrillation, the aorta was crossclamped. A few seconds after crossclamping of the aorta, at a core temperature of $32^{\circ} \mathrm{C}$, sudden bilateral mydriasis developed, suggesting impaired cerebral perfusion. At the same time, the mean right radial artery pressure dropped suddenly. A self-inflating balloon cannula was inserted high into the internal jugular vein via the right atrium. The cannula was then connected with an arterial line to the heart-lung machine. A retrograde cerebral perfusion with oxygenated blood flowing in a retrograde direction through the jugular vein and thus to the brain was begun. The retrograde cerebral blood flow was maintained at 200 to $250 \mathrm{ml} / \mathrm{min}$ with a mean pressure of $25 \mathrm{~mm} \mathrm{Hg}$ while the patient was cooled, the aortotomy performed, the aortic valve resuspended, and the proximal aortic anastomosis completed (45 minutes). Then, during deep hypothermic circulatory arrest, the aortic clamp was released and the distal aorta closely inspected. A single intimal tear was present $2 \mathrm{~cm}$ above the coronary ostia; no intimal tears were present in the aortic arch. Good back blood flow from the left common carotid artery and the innominate artery was evident. During the period of deep hypothermic $\left(18^{\circ} \mathrm{C}\right)$ circulatory arrest ( 36 minutes), the arch anastomosis was performed. Blood samples simultaneously drawn from a side port of the cerebral perfusion cannula (arterial inflow) and from the back bleeding from the left carotid and innominate arteries (venous outflow) at the beginning of circulatory arrest were sent for arterial blood gases measurements. The results showed an oxygen extraction of $3.3 \mathrm{ml} / \mathrm{dl}$ throughout the brain. The postoperative course was uneventful and the patient awoke 6 hours after the operation. The way in which she awoke and behaved was within normal limits.

Encouraged by reported data, ${ }^{4}$ we have recently been using retrograde cerebral perfusion during hypothermic circulatory arrest, and we are impressed by our good results. Therefore, faced with the sudden appearance of cerebral malperfusion, we thought that any neurologic sequelae could be prevented by a selective retrograde perfusion of the brain through the superior 
vena cava. The presence of cerebral malperfusion was suspected only on the basis of clinical findings; we do not have any direct anatomic evidence, as elegantly demonstrated by Neustein and associates. ${ }^{2}$ Nevertheless, we think that the sudden occurrence of bilateral mydriasis associated with a fall in the right radial pressure at the time of aortic crossclamping is strongly suggestive of an impaired cerebral perfusion. Furthermore, monitoring of right radial versus femoral artery pressure is the most commonly used, although indirect, method to unveil a deficit of brain perfusion. A delay in correcting this anatomic condition diagnosed simply on the basis of this indirect method has been reported to lead to cerebral deficit or death. ${ }^{1}$

We cannot state, on the basis of this clinical case, that the good surgical outcome is ascribed only to the use of retrograde cerebral perfusion before and during the period of circulatory arrest. Nonetheless, we can consider that our patient underwent 45 minutes of a somewhat impaired cerebral perfusion when the body temperature was well above the degree recommended for safe cerebral protection, followed by 36 minutes of total circulatory arrest at a temperature of $18^{\circ} \mathrm{C}$. Given the patient's good outcome, it is possible that a cerebral perfusion sufficient for the brain's metabolic needs was always assured throughout the surgical procedure. Doubts remain regarding the retrograde cerebral blood path during the period preceding circulatory arrest. The cerebral venous outflow cannot be drained from the left carotid and innominate arteries, as during the period of circulatory arrest when the aortic arch is wide open. Then, given the fact that jugular venous pressure was always constant at values of $20 \mathrm{~mm} \mathrm{Hg}$ during the retrograde perfusion, we can assume that the orly cerebral drainage path was through the left jugular venous system. This latter aspect will need to be resolved if the reproducibility of the method is to be assessed.

If the findings herein reported can be confirmed in the future, retrograde cerebral perfusion could be considered another technical therapeutic option when cerebral malperfusion, occurring during aortic surgery, needs to be promptly corrected. This experience may help to provide some insights to the understanding of the efficacy and safety of retrograde cerebral perfusion during circulatory arrest.

Ruggero De Paulis, $M D$ Dionisio F. Colella, $M D$

Carlo Bassano, $M D$

Alessandro Ricci, $M D$

Luigi Chiariello, $M D$

Departments of Cardiac Surgery and Anesthesiology Tor Vergata University of Rome Rome, Italy

\section{R EFERENCES}

1. Borst HG, Laas $\mathbf{J}$, Heinemann $\mathbf{M}$. Type A aortic dissection: diagnosis and management of malperfusion phenomena. Semin Thorac Cardiovasc Surg 1991;3:238-41.
2. Neustein SM, Lansman SL, Quintana CS, Suriani R, Ergin A, Griepp RB. Transesophageal Doppler echocardiographic monitoring for malperfusion during aortic dissection repair. Ann Thorac Surg 1993;56:358-61.

3. Parr GVS, Manley NJ, Williams DR, et al. Obstruction of the true lumen during retrograde perfusion of type I aortic dissection: a simplified solution. Ann Thorac Surg 1980; 30:495-8.

4. Safi HJ, Brien $\mathrm{HW}$, Winter JN, et al. Brain protection via cerebral retrograde perfusion during aortic arch aneurysm repair. Ann Thorac Surg 1993;56:270-6.

\section{Potential uses of prostaglandin $E_{1}$ analog for cardiovascular disease}

\section{To the Editor:}

We read with interest two recent reports in the JOURNAL regarding the therapeutic efficacy of prostaglandin $\mathrm{E}_{1}\left(\mathrm{PGE}_{1}\right)$ for improved preservation of ultrastructure in heart-lung transplantation ${ }^{1}$ and amelioration of pulmonary function in patients with heart and lung failure after cardiotomy. ${ }^{2} \mathrm{PGE}_{1}$ analogs, specifically misoprostol (Cytotec), are already in use for inhibition of acid secretion in patients with peptic ulceration. These analogs differ from $\mathrm{PGE}_{1}$ and $\mathrm{PGE}_{2}$ in that there is a methylydroxyl group at position 16 and the absence of the cis double bond at position 5 . These analogs, moreover, are associated with a longer survival for cardiac allografts in murine models ${ }^{3}$ and prevention of acute renal graft rejection in human beings. ${ }^{4}$ Such immunologicaly based mechanisms, we hypothesized, may be related to downregulation of cytokine production by effector cells such as macrophages. To test this hypothesis, we treated peritoneal exudative cells from mice (which are predominantly macrophages) with lipopolysaccharide, an endotoxin known to activate these cells and cause them to produce tumor necrosis factor, interferon gamma, and interleukin-1 and interleukin-6, in the presence or absence of a $\mathrm{PGE}_{1}$ analog (provided by Searle Pharmaceutical, Chicago, Ill.). As seen in Table I, a dose-dependent decrease in cytokine production occurred with the addition of the analog. Furthermore, in other experiments, the misoprostol protected tumor necrosis factor-sensitive cells from that cytokine.

We theorize that some of the effects seen with the amelioration of lung function in the transplant situation, as well as after cardiotomy, may be due to downregulation of these potentially toxic cytokines. The $\mathrm{PGE}_{1}$ would prevent activated alveolar macrophages from producing harmful levels of these substances, which are associated with endothelial injury, edema, and eventually fibrosis. If the commercially available analog works in the same manner as the $P G E_{1}$, it could be potentially useful not only in future trials of organ preservation, but as a potential

Table I. Influence of $P G E_{1}$ analog (misoprostol) on cytokine production by peritoneal exudative cells

\begin{tabular}{lcccccc}
\hline & \multicolumn{7}{c}{ Misoprostol $(\mu \mathrm{g} / \mathrm{ml})$} \\
\cline { 2 - 7 } & 0 & 0.156 & 0.313 & 1.25 & 2.5 & 5 \\
\hline TNF $(\mathrm{ng} / \mathrm{ml})$ & $1.72 \pm 0.36$ & $0.87 \pm 0.21^{*}$ & $0.64 \pm 0.17^{*}$ & $0.50 \pm 0.14^{*}$ & $0.39 \pm 0.17^{*}$ & $0.27 \pm 0.14^{*}$ \\
IL-1 $(\mathrm{pg} / \mathrm{ml})$ & $186 \pm 19$ & $122 \pm 22$ & $119 \pm 18^{*}$ & $111 \pm 25^{*}$ & $78 \pm 22^{*}$ & $80 \pm 12^{*}$ \\
IL-6 $(\mathrm{pg} / \mathrm{ml})$ & $3502 \pm 479$ & $932 \pm 271^{*}$ & $781 \pm 289^{*}$ & $989 \pm 323^{*}$ & $780 \pm 215^{*}$ & $582 \pm 230^{*}$ \\
Survival (\%) & 100 & $101 \pm 1$ & $97 \pm 2$ & $91 \pm 2$ & $91 \pm 3^{*}$ & $90 \pm 2$ \\
\hline
\end{tabular}

$T N F$, Tumor necrosis factor; $I L$, interleukin.

${ }^{*} P_{2}<0.05$ from 0 misoprostol. 